\title{
A novel family of diversified immunoregulatory receptors in teleosts is homologous to both mammalian Fc receptors and molecules encoded within the leukocyte receptor complex
}

\author{
James L. Stafford • Eva Bengtén • Louis Du Pasquier • \\ Robin D. McIntosh • Sylvie M. Quiniou • L. W. Clem • \\ Norman W. Miller • Melanie Wilson \\ Received: 19 April 2006 / Accepted: 23 May 2006 / Published online: 15 July 2006 \\ (C) Springer-Verlag 2006
}

\begin{abstract}
Three novel and closely related leukocyte immune-type receptors (IpLITR) have been identified in channel catfish (Ictalurus punctatus). These receptors belong to a large polymorphic and polygenic subset of the Ig superfamily with members located on at least three independently segregating loci. Like mammalian and avian innate immune regulatory receptors, IpLITRs have both putative inhibitory and stimulatory forms, with multiple types coexpressed in various lymphoid tissues and clonal leukocyte cell lines. IpLITRs have an unusual and novel relationship to mammalian and avian innate immune receptors: the membrane distal Ig domains of an individual IpLITR are related to fragment crystallizable receptors (FcRs) and FcR-like proteins, whereas the membrane
\end{abstract}

Electronic supplementary material Supplementary material is available for this article at $\mathrm{http}: / / \mathrm{dx}$.doi.org/10.1007/s00251006-0134-1 and is accessible for authorized users.

GenBank Submissions: The sequences presented in this article have been submitted to GenBank under the following accession numbers: AAW82352, IpLITR1; AAW82353, IpLITR2; AAW82354, IpLITR3.

J. L. Stafford · E. Bengtén · R. D. McIntosh · L. W. Clem •

N. W. Miller $\cdot$ M. Wilson $(\triangle)$

Department of Microbiology,

University of Mississippi Medical Center,

2500 North State Street,

Jackson, MS 39216-4505, USA

e-mail: mwilson@microbio.umsmed.edu

L. Du Pasquier

Institute of Zoology, University of Basel,

Basel, Switzerland

S. M. Quiniou

USDA-ARS/CGRU,

141 Experimental Station Road,

Stoneville, MS 38701, USA proximal Ig domains are related to several leukocyte receptor complex encoded receptors. This unique composition of Ig domains within individual receptors supports the hypothesis that functionally and genomically distinct immune receptor families found in tetrapods may have evolved from such ancestral genes by duplication and recombination events. Furthermore, the discovery of a large heterogeneous family of immunoregulatory receptors in teleosts, reminiscent of amphibian, avian, and mammalian Ig-like receptors, suggests that complex innate immune receptor networks have been conserved during vertebrate evolution.

Keywords Evolution · Molecular immunology · Inhibitory receptors $\cdot$ Innate immunity $\cdot$ Teleosts

\section{Introduction}

Immune receptors that bind similar ligands often form clusters that are encoded on distinct chromosomes in the human genome. For example, receptors that bind to the Fc portion of Ig [fragment crystallizable receptors (FcRs)] are found on chromosome 1q21-23 and consist of the highaffinity IgG and IgE receptors (Fc $\gamma \mathrm{RI}$ and FceRI) and the related low- or moderate-affinity Ig receptors (Fc $\gamma$ RII, Fc $\gamma$ RIII, and Fc $\gamma$ RIV) (Hullet et al. 1991; Ravetch and Kinet 1991; Hullet and Hogarth 1994; Daeron 1997; Falk et al. 2005; Falk and Ravetch 2006). Alternatively, immune receptors involved in the recognition of major histocompatability class 1 (MHC I) antigens, such as killer cell Ig-like receptors (KIRs) and leukocyte Ig-like receptors (LILRs), are clustered together on chromosome $19 \mathrm{q} 13.4$ in a region known as the leukocyte receptor complex (LRC) (Kremer et al. 1992; Wagtmann et al. 1997; Wende et al. 1999; 
Martin et al. 2002). Recently, comparative studies have suggested that FcRs and LRC-encoded receptors, although genomically and functionally distinct, appeared to have evolved from a common ancestor. This contention is supported in part by recent attempts to find LRC regions in other species.

Mouse chromosome 7 is syntenic to the human LRC and encodes the paired Ig-like receptors (PIRs) that were originally discovered in an attempt to find a murine $\operatorname{IgA}$ receptor ortholog (CD89; Kubagawa et al. 1997; Hayami et al. 1997). Subsequently, searches for PIR relatives resulted in the identification of the chicken Ig-like receptors (CHIRs) in birds (Dennis et al. 2000), representing a multigene family encoded within an avian LRC-like region (Nikolaidis et al. 2005a; Viertlboeck et al. 2005). More importantly, CHIR Ig domain sequences are not only related to the mammalian PIRs and KIRs, but also to various FcR Ig domains (Dennis et al. 2000). This previously unrecognized relationship was supported by phylogenetic and comparative structural analysis and was the first evidence suggesting that certain genomically and functionally diverse mammalian and avian immune receptor families probably evolved from a common ancestor (Dennis et al. 2000; Nikolaidis et al. 2005b).

Compared to mammals and birds, bony fish are among the most phylogenetically distant vertebrates with characteristic innate and adaptive immune systems that have mammalian-like B and $\mathrm{T}$ cells (Yoder 2004). Furthermore, due to the establishment of clonal lymphoid cell lines, catfish are known to also possess natural killer (NK)-like cells capable of killing xenogeneic targets by an antibody-dependent cell-mediated cytotoxicity (ADCC) process (Shen et al. 2002-2004). This functional evidence suggested that catfish NK cells possess a putative $\mathrm{Fc} \mu \mathrm{R}$ (Shen et al. 2003). Additionally, catfish NK cells can kill allogeneic targets in an ADCC-independent manner, indicating that target recognition may also involve KIR and/or LILR-like receptors (Shen et al. 2002, 2004). However, to date, FcR and/or KIR/LILR homologs have not been described in ectothermic vertebrates, making it difficult to elucidate the evolutionary history of these important receptor families. Large families of putative activatory and inhibitory novel immune-type receptors (NITRs) also belonging to the Ig superfamily (IgSF) have been described in teleosts. Pufferfish (Spheroides nephelus), zebrafish (Danio rerio), catfish, and rainbow trout (Oncorhynchus mykiss) all express NITRs (Strong et al. 1999; Yoder et al. 2001, 2002; Hawke et al. 2001). Because some NITRs are also expressed by catfish NK cells (Hawke et al. 2001), it has been suggested that these receptors may represent the "functional orthologs" of the mammalian KIRs and/or LILRs (Yoder et al. 2001; Hawke et al. 2001). However,
NITRs do not exhibit any sequence identity or phylogenetic relationship to such receptors (Yoder et al. 2001) and have only been identified in bony fishes.

Herein is described a novel set of IgSF receptors present in the channel catfish, and possibly other teleosts, that are composed of Ig domains related to functionally diverse immunoreceptor families present in mammals. Detailed sequence analysis supports that individual catfish leukocyte immune-type receptors (IpLITRs) contain a unique composition of Ig C2-like domains sharing homology with both FcRs and receptors encoded within the LRC. Thus, IpLITRs provide further evidence supporting the common evolutionary origins of genomically and functionally distinct immunoregulatory receptor families present in mammals.

\section{Materials and methods}

Experimental animals and cell lines

Catfish (1-2 kg) were obtained from a commercial source (ConAgra, Isola, MS, USA) and maintained in individual tanks as described previously (van Ginkel et al. 1992). Catfish peripheral blood leukocytes (PBL) were isolated from heparinized blood by centrifugation on a cushion of FicollHypaque (Lymphoprep, Accurate Chemical, Westbury, NY, USA) as described previously (Miller et al. 1994a). Catfish cells lines were grown at $27^{\circ} \mathrm{C}$ in AL-3 medium consisting of equal parts AIM-V and L-15 (Life Technologies, Gaithersburg, MD, USA) adjusted to catfish tonicity with $10 \%(\mathrm{v} / \mathrm{v})$ deionized water and supplemented with $1 \mu \mathrm{g} / \mathrm{ml}$ $\mathrm{NaHCO}_{3}, 50 \mathrm{U} / \mathrm{ml}$ penicillin, $50 \mu \mathrm{g} / \mathrm{ml}$ streptomycin, $20 \mu \mathrm{g} / \mathrm{ml}$ gentamicin, $50 \mathrm{uM}$ 2-ME, and 3\% heat-inactivated, pooled, normal catfish serum (Miller et al. 1994a,b). 3B11 is a cloned autonomous B cell line generated from an outbred catfish by mitogen stimulation (Wilson et al. 1997). $\mathrm{G} 14 \mathrm{D}$ is an automonous $\mathrm{T}$ cell line derived from a gynogenetic catfish (Hogan et al. 1999), whereas 42TA is a macrophage cell line derived from a different outbred fish (Miller et al. 1994b). TS32.15 and TS32.17 are clonal nonautonomous antigen-dependent cytotoxic $\mathrm{T}$ cell lines developed from an alloantigen immunized outbred fish (Stuge et al. 2000). 1F3 is a clonal nonautonmous NK-cell line developed from an alloantigen-stimulated culture of PBL from a naive outbred fish (Shen et al. 2004). The nonautonomous cytotoxic $\mathrm{T}$ cell and NK cell lines require weekly restimulation with irradiated allogeneic cells for continuous proliferation. The channel catfish ovary fibroblast cell line (CCO; Browser and Plumb 1980) was provided by Dr. V.G. Chinchar (University of Mississippi Medical Center). CCO cells were maintained in Dulbecco's modified Eagle's medium (Sigma-Aldrich, St. Louis, MO, USA) supplemented with $10 \%$ FCS. 
Channel catfish LITRs and sequence analysis

In a search for IgSF receptors expressed by alloantigen stimulated catfish PBL and various catfish clonal cell lines, several expressed sequence tag (EST) libraries were analyzed. Subsequently, IpLITR1 and IpLITR3 cDNAs were identified by single pass sequencing of a cDNA library generated from a catfish 7-day-old mixed lymphocyte culture (MLC) enriched in NK-like cells, MLC52-1 (Shen et al. 2004; Stuge et al. 1997). IpLITR2 was obtained from the catfish 42TA macrophage cell line (Miller et al. 1994b) cDNA library. The cDNAs were sequenced on both strands using universal forward and reverse primers and genespecific primers. Nucleotide and amino acid sequences were analyzed using DNASTAR software (Madison, WI, USA) and aligned using CLUSTALW (Thompson et al. 1997). Neighbor-joining (NJ) trees with pairwise gap deletions were drawn using MEGA v3.0 (Kumar et al. 2001). Maximum parsimony and maximum likelihood trees were drawn with essentially the same results (data not shown). Similarity searches were performed with the extracellular Ig domains of IpLITR1, IpLITR2, and IpLITR3 using position-specific iterative (PSI) and Basic Local Alignment Search Tool (BLAST) analysis (Altschul et al. 1990) against the National Center for Biotechnology Information (NCBI) nonredundant database. SOSUI (signal) beta version, Simple Modular Architecture Research Tool (SMART) (Letunic et al. 2004), Pfam databases (Bateman et al. 2004) and the threedimensional position-specific scoring matrix server (Kelly et al. 2000) were used for predictions of signal peptides, Ig domains, transmembrane (TM) segments, and secondary structure. Sequence decorations were performed using GeneDoc (http://psc.edu/biomed/genedoc).

Southern and Northern blot analyses

Genomic DNA was prepared from erythrocytes of 16 catfish siblings. These siblings were representatives of a 78-member family obtained from the Catfish Genetics Research Unit, United States Department of Agriculture, Agricultural Research Service (Stoneville, MS, USA; designated family 1 ). The sibling catfish $1-16$ used were A10, A5, A32, A22, A38, A36, A16, A29, A18, A49, A159, A15, A23, A25, A186, and A37, respectively (Quiniou et al. 2005). The DNA $(10 \mu \mathrm{g})$ was digested to completion with Pst1 or EcoRI, separated on 1\% agarose gels and transferred by capillary action onto Hybond-N+ membranes (Amersham Pharmacia Biotech, Arlington Heights, IL, USA) using standard techniques. Hybridizations were performed in Rapid-hyb buffer (Amersham Pharmacia Biotech) at $65^{\circ} \mathrm{C}$ and membranes were washed at high stringency $\left(65^{\circ} \mathrm{C}\right.$ with $0.1 \times$ saline-sodium citrate, $0.1 \%$ sodium dodecyl sulfate). Specific probes (Table 1) were amplified by PCR using IDPol DNA polymerase (ID Labs Biotechnology, London, Ontario, Canada) according to the manufacturer's recommended protocol. Typical parameters were: $1 \mathrm{~min} 94^{\circ} \mathrm{C}$, followed by 29 cycles of $94^{\circ} \mathrm{C} 30 \mathrm{~s}, 62^{\circ} \mathrm{C} 30 \mathrm{~s}, 72^{\circ} \mathrm{C} 1 \mathrm{~min}$, then extension at $72^{\circ} \mathrm{C}$ for $10 \mathrm{~min}$. Probes were random primed labeled with $\left[{ }^{32} \mathrm{P}\right]$ $2^{\prime}$-deoxycytidine 5'-triphosphate using a Megaprime labeling kit (Amersham Pharmacia Biotech).

Total RNA from catfish PBL and various tissues was prepared using RNA-Bee (Tel-test, Friendswood, TX, USA); $10 \mu \mathrm{g}$ of each sample were electrophoresed on $1.5 \%$ formaldehyde-agarose gels and transferred by capillary action onto Hybond-N+ (Amersham Pharmacia Biotech), hybridized, and washed as above.

\section{Reverse transcription (RT)-PCR}

Total RNA from PBL, MLC, various tissues, and catfish clonal cell lines were prepared as described above, and treated with DNase I (Invitrogen Life Technologies, Carlsbad, CA, USA) according to the manufacturer's recommended protocol. One $\mu \mathrm{g}$ RNA was reverse transcribed using an oligo-T primer and 200 units of Superscript III reverse transcription (RT) (Invitrogen Life Technologies). RT-PCR was performed using specific primers for IpLITR and the housekeeping gene elongation factor 1 alpha (IpEF1 $\alpha$; Table 1). Typical parameters were: $3 \min 94^{\circ} \mathrm{C}$, followed by 30 cycles of $94^{\circ} \mathrm{C} 45 \mathrm{~s}, 58^{\circ} \mathrm{C} 45 \mathrm{~s}$, $72^{\circ} \mathrm{C} 2 \mathrm{~min}$, then extension at $72^{\circ} \mathrm{C}$ for $10 \mathrm{~min}$. PCR products were visualized on $1.2 \%$ Tris-acetate-EDTA agarose gels. PCR products were also cloned into pCR4-

Table 1 IpLITR PCR primers used for generating Ig domain-specific probes and gene-specific primers used in RT-PCR experiments

\begin{tabular}{lll}
\hline Primer & & \\
\hline IpLITR1 & Forward & 5' GTCGGACAAGGTCAAGTTCTG 3' \\
D1 & Reverse & 5' GGCTTTTGGTCTCTCTATCAC 3' \\
IpLITR1 & Forward & 5' AATCCTGATGAACAGGTGTACC 3' \\
D4 & Reverse & 5' GTGTTTACAGTGGTAGAAACC 3' \\
IpLITR2 & Forward & 5' AGTCGTGAAGGAGCTGTACTGA 3' \\
D3 & Reverse & 5' TTCACTGCCAGAATGATGGTCAC 3' \\
IpLITR3 & Forward & 5' CTGAGGGACATCCTCTGACCTT 3' \\
D6 & Reverse & 5' GTGTTTACAGTGGTAGAAACC 3' \\
IpLITR1 & Forward & 5' GTCGGACAAGGTCAAGTTCTG 3' \\
GSP & Reverse & 5' CTGCAGACATGATGAACTTCT 3' \\
IpLITR2 & Forward & 5' GTCGGACAAGCTCAAGAGTTT 3' \\
GSP & Reverse & 5' TGTGTAGTAGAGTGGGTTTCC 3' \\
IpLITR3 & Forward & 5' GTCGGACAAGGTCAAGTTCTG 3' \\
GSP & Reverse & 5' GCTTTTGGGTGACTCTCCTCT 3' \\
IpEF1 $\alpha$ & Forward & 5' GACTGCCACACTGCTCACATTG 3' \\
GSP & Reverse & 5' TTAGTTACTCAGCAGCTTTCTTCC \\
\hline
\end{tabular}

GSP gene-specific primers 


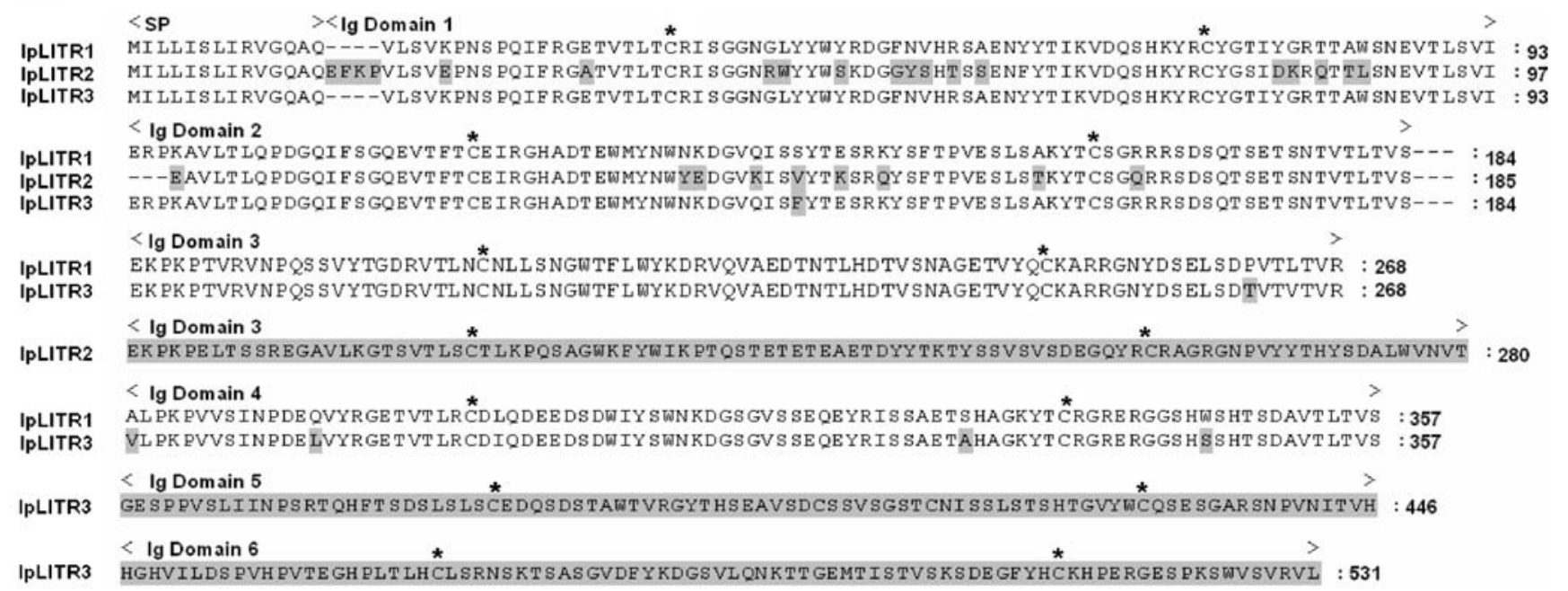

\section{b}

C

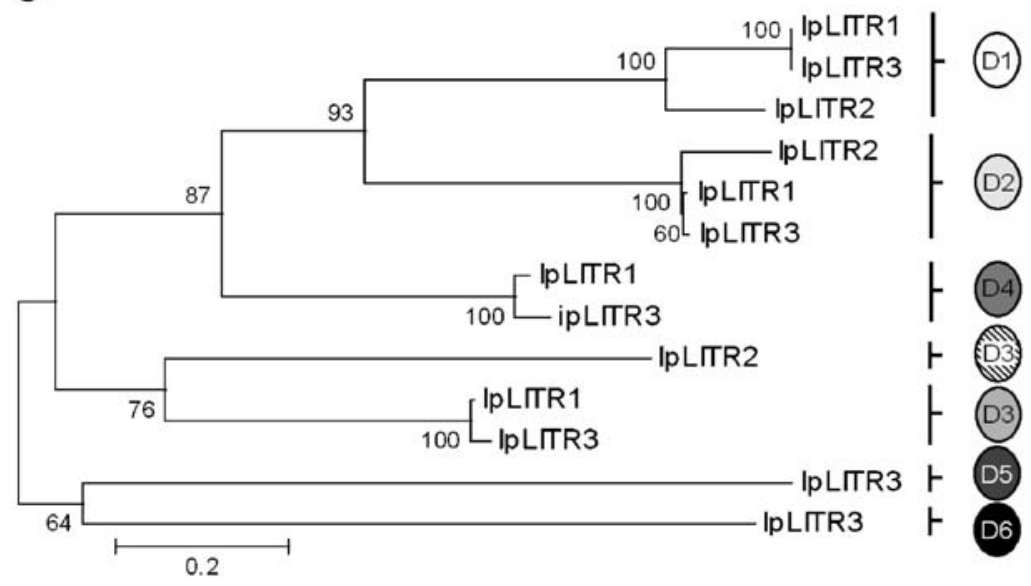

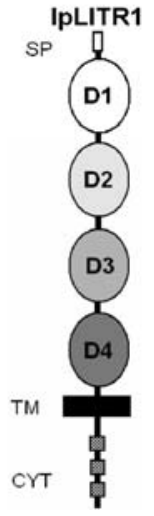

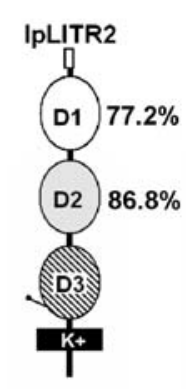

IPLITR2 -DVKAPLSVLKLLSSAVAASPYVLVTIILAVKCYRARTDPDEDNRPYRVIEAEASV : 335

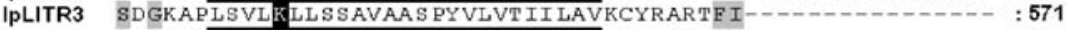

Fig. 1 Predicted amino acid sequence, domain comparisons, and schematic representation of IpLITR1, IpLITR2, and IpLITR3. a Alignment of the extracellular and $\mathbf{b}$ TM/CYT regions of IpLITR1, IpLITR2, and IpLITR3. Signal peptide $(S P)$, and immunoglobulin (Ig) domains are labeled; cysteine residues predicted to be involved in intrachain disulfide bonds are marked with asterisks; gray shaded residues represent differences from IpLITR1 in a and differences from IpLITR2 in b. TMs are underlined, ITIM-like motifs are boxed, an overlapping ITSM within the CYT of IpLITR1 is indicated by a bracket, and TM charged residues are shaded black and marked $(+)$. c Phylogenetic analysis of Ig domains in IpLITRs.NJ trees with pairwise gap deletions were drawn using MEGA v3.0 (Kumar et al. 2001) with 10,000 bootstrap replications, and bootstrap values $>50 \%$ are shown. Branch lengths were measured in terms of amino acid substitutions and a scale bar is shown below the trees. The predicted SP, Ig domains, TM, and CYT are indicated. ITIM-like motifs are shown as boxes, N-linked glycosylation sites are marked as ballpoint lines. Individual IpLITR domains are shaded according to their relatedness between IpLITRs and percent amino acid identity with IpLITR1 Ig domains indicated to the left of IpLITR2 and IpLITR3. IpLITR2 D3 and IpLITR3 D5 and D6 are $15.2-39.3 \%$ identical to all IpLITR1 Ig domains 
a

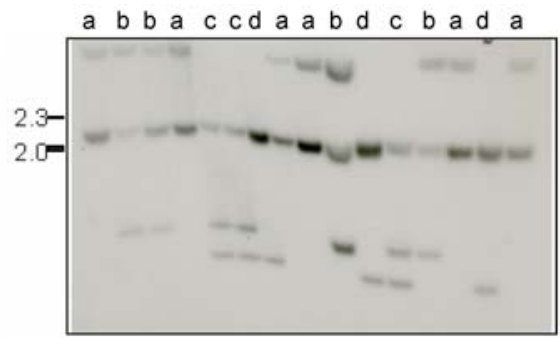

b

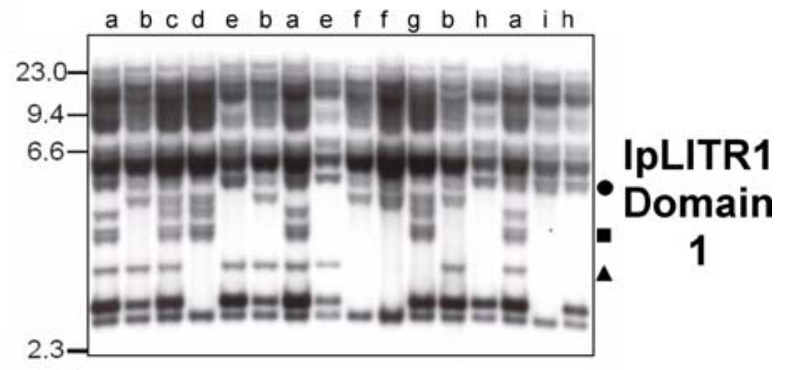

C

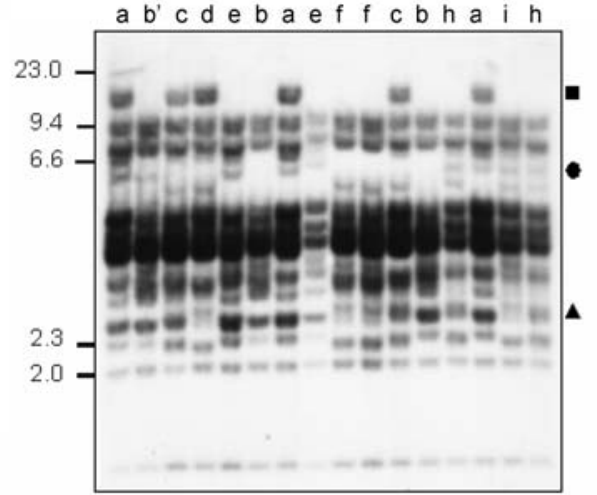

IpLITR1 Domain 4

d

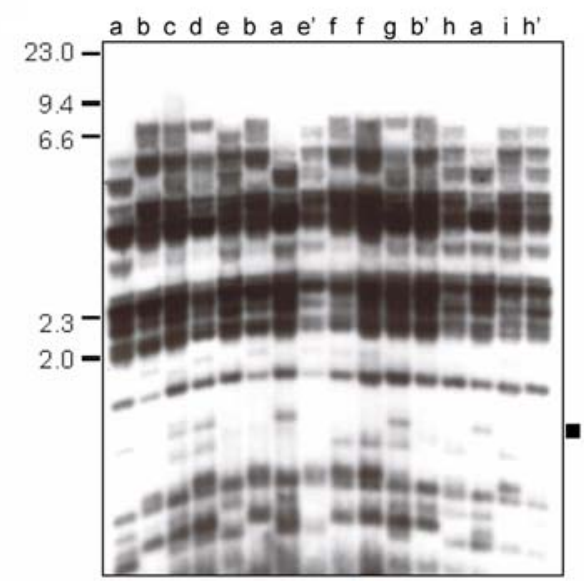

IpLITR3 Domain 6

Fig. 2 Southern blot analyses. Genomic DNA from 16 sibling catfish was digested, separated, transferred to nylon membranes, and hybridized with a MHCII $\beta$, b IpLITR1 D1, c IpLITR1 D4, and d IpLITR3 D6-specific probes. Letters $a-i$ indicate different segregation patterns $\left(b^{\prime}, e^{\prime}\right.$, and $h^{\prime}$ represent variant segregation patterns that may be the result of recombination). Representative RFLP bands illustrating different linkage groups are indicated by $\bullet, \boldsymbol{\Delta}$, and $\boldsymbol{\square}$ Kilobase size markers are indicated to the left of each blot

TOPO $^{\circledR}$ (Invitrogen Life Technologies) and verified by sequencing. The Genbank accession number for IpEF $1 \alpha$ is CB938718.

\section{Results}

Catfish LITR sequences

In a search for IgSF receptors expressed by alloantigenstimulated catfish PBL and various catfish clonal cell lines, several EST libraries were analyzed. Three IpLITR sequences were subsequently identified that encoded type I TM proteins with extracellular C2-like Ig domains, which were predicted using SMART (see Electronic Supplementary Material). Even though these sequences vary in length, they are highly similar (Fig. 1a,b). All three receptors have identical signal peptides and IpLITR1 and IpLITR3 have almost identically encoded Ig domains D1, D2, D3, and D4 (Fig. 1a). Comparatively, IpLITR2 D1 and D2 are 77.2 and $86.8 \%$ identical at the amino acid level to their IpLITR1 and IpLITR3 counterparts (Fig. 1c). IpLITR1 encodes a 346 amino acid extracellular region consisting of four Ig domains, a 23-amino acid TM segment and a 116-amino acid cytoplasmic tail (CYT). The IpLITR1 CYT contains two immunotyrosine-based inhibition motifs (ITIMs) centered at Y439 and Y461 and an ITIM-like motif (SEYTTE) centered at Y479 (Fig. 1b) (Daeron and Vivier 1999; Ravetch and Lanier 2000; Billadeau and Leibson 2002). An overlapping immunotyrosine-based switch motif (ITSM; TVYSQL) centered at Y465 is also present in the CYT of IpLITR1 (Shlapatska et al. 2001). In contrast, the smaller IpLITR2 and the longer IpLITR3 transcripts encode for molecules with identical 25-amino acid TMs containing a lysine residue and very similar charged CYTs (Fig. 1b). Overall, the individual IpLITRs are composed of a membrane distal to membrane proximal ordering of Ig domains, each containing similar D1s and D2s. However, the membrane proximal domains vary, i.e., IpLITR3 D5 and D6 are only $15.7-24.7 \%$ and $15.2-25 \%$ identical, respectively, to all other IpLITR Ig domains, and IpLITR2 D3 is only $17.9-39.3 \%$ identical to other IpLITR domains (Fig. 1c).

IpLITRs belong to polymorphic and polygenic gene families

Sibling restriction fragment length polymorphism (RFLP) patterns using domain-specific probes for the various

Table 2 Identification of IpLITR-like genes in the zebrafish genome

\begin{tabular}{ll}
\hline Accession numbers & Chromosome \\
\hline BX005156 & 8 \\
BX000524 & 8 \\
BX511176 & 3 \\
BX649627 & 3 \\
CAAK01000490.1 & 7 \\
\hline
\end{tabular}

Search $(04 / 05)$ of the zebrafish whole genome shotgun database of the NCBI (Sanger Genomic Institute) 
Fig. 3 Northern blot and RTPCR analyses of IpLITR tissue expression. Catfish LITR tissue expression was performed by Northern blot analysis using a an IpLITR1 D1-specific probe, and b an IpLITR2 D3specific probe. Total RNA from pronephros (head kidney), mesonephros (trunk kidney), spleen, heart, liver, gill, and muscle were examined.

Kilobase markers are on the left margin and arrows indicate the major hybridizing bands observed. RNA integrity and load levels were determined by hybridization using a catfish $\mathrm{EF} 1 \alpha$ probe as a housekeeping gene. c RT-PCR analyses of IpLITR1 and IpLITR2 in various catfish tissues. The sizes of the IpLITR bands verified by sequencing are indicated at the right margin and base pair sizes are at the left margin a

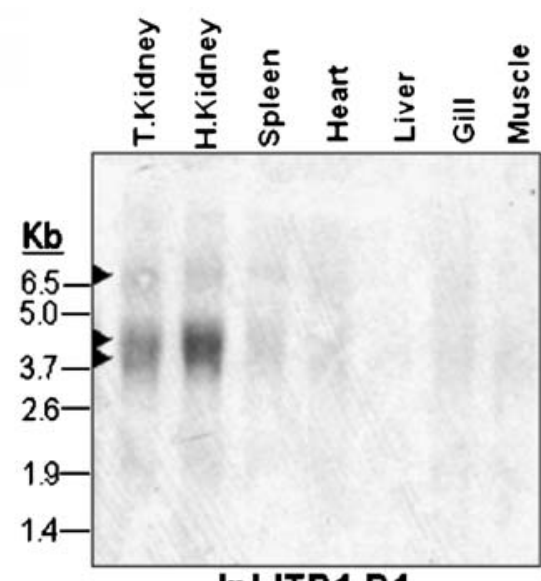

IpLITR1 D1

b

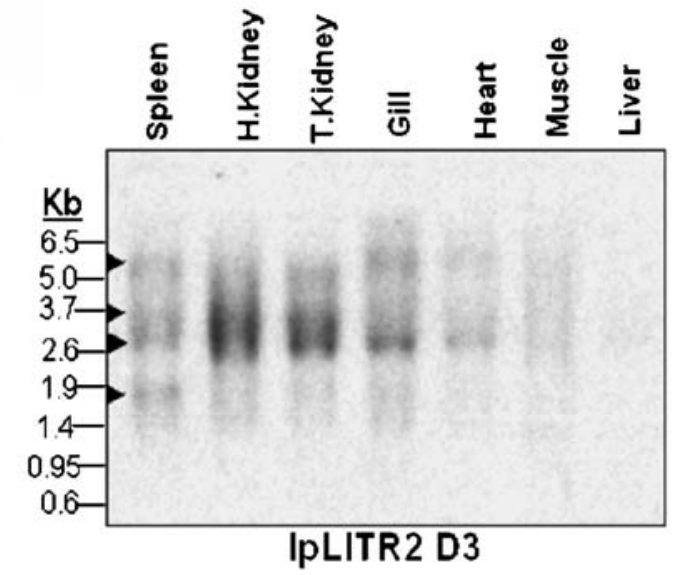

IpEF1 $\alpha$

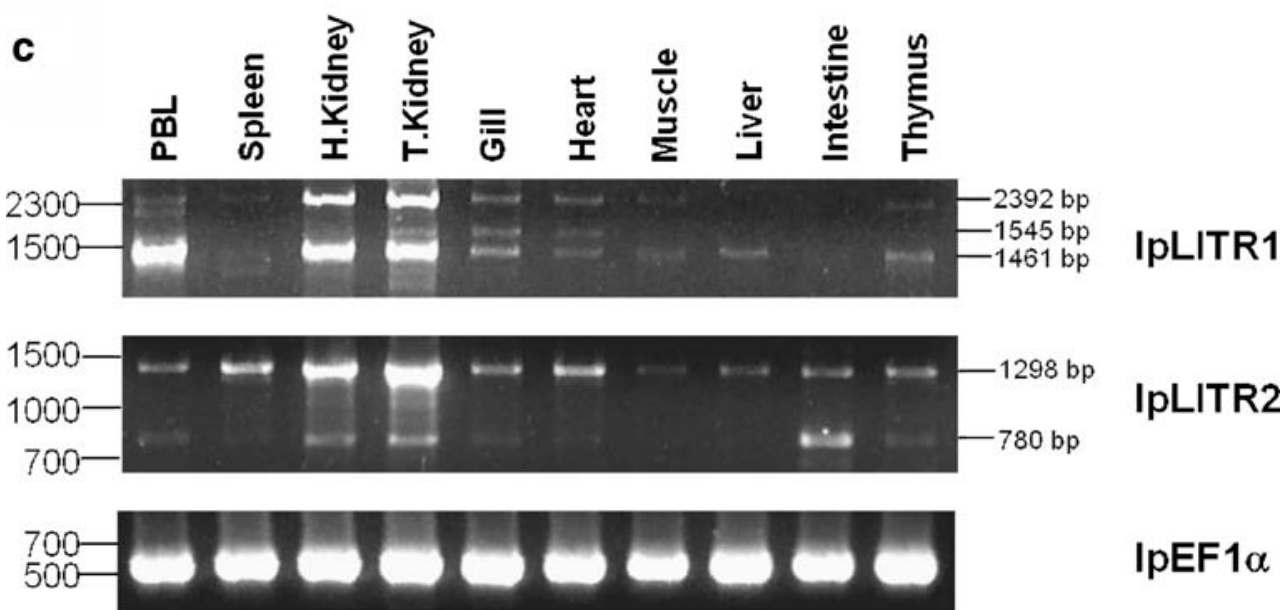

IpLITRs show a large number of hybridizing bands with each probe used, as well as differences in the banding patterns among siblings (Fig. 2). These findings indicate that the IpLITR gene complex is both polygenic and poly- morphic. However, it is presently unknown whether these polymorphic differences are due to variability within the exons encoding these receptors and/or within the introns. Some of the high-intensity bands found in each of the 
Southern blots may reflect multiple IpLITR copies, further increasing the possible number of genes. Importantly, the Southern blot segregation patterns show that there is more than one IpLITR locus. For example, among the 16 sibling fish used there are at least nine, perhaps more, different RFLP patterns for IpLITR D1 (Fig. 2b). If these genes were closely linked then only four RFLP patterns (barring crossover events) would be observed, as shown for the MHC class II $\beta$ RFLP patterns (Fig. 2a). When all Southern blots are compared, at least three distinct linkage groups (marked by $\bullet, \mathbf{\Lambda}$, and $\mathbf{-}$ are observed, indicating the presence of multiple independently segregating loci, and none of these are linked to the MHC II RFLP (Fig. 2b-d). Similar results were also obtained using the IpLITR2 D3 probe (data not shown). Mining of the zebrafish genome for IpLITR relatives [whole genome shotgun database of the NCBI (Sanger Genomic Institute, Cambridge, UK)] further supports that these receptors are present on different chromosomes because five contigs were identified with IpLITR-like homologs on zebrafish chromosomes 3, 7, and 8 (Table 2).

\section{IpLITR expression}

Northern blot analyses using a cross hybridizing IpLITR1 D1 probe revealed that LITR message is highly expressed in the hematopoietic pronephros and mesonephros, with predominant transcript sizes of $\sim 6.6, \sim 4.0$, and $\sim 3.8 \mathrm{~Kb}$ (Fig. 3a). Low levels of IpLITR D1 expression were detected in the spleen, with little to no message detected in the heart, liver, gill, or muscle. The use of the more specific IpLITR2 D3 probe revealed message expression not only in the pronephros and mesonephros, but also in the spleen, gill, and perhaps heart with transcripts of $\sim 6.0$, $\sim 3.7, \sim 2.7$, and $\sim 1.9 \mathrm{~Kb}$ (Fig. 3b). RNA load levels for each of the tissues tested were similar based upon hybridization with a catfish $\mathrm{EF} 1 \alpha$ probe. RT-PCR, using primers specific for the putative inhibitory IpLITR1 and the putative activating IpLITR2 as representative IpLITRs, was also used to assess tissue expression. As shown in Fig. 3c, IpLITR1 and IpLITR2 are in most cases coordinately expressed in a variety of different tissues and confirm the Northern blot data. In addition, multiple PCR products were amplified using the IpLITR1 and IpLITR2 specific primers, which is reminiscent of the multiple transcripts observed following RNA blotting. Because the predicted sizes of PCR products for the prototype IpLITR 1 and IpLITR2 are 1,461 and $780 \mathrm{bp}$, respectively, at least two additional IpLITR 1 products $(1,545$ and 2,392 bp) and one additional IpLITR2 product (1,298 bp) were amplified (Fig. 3c).

To further examine the expression of IpLITR1 and IpLITR2, RT-PCR was performed using RNA from catfish clonal macrophage, B, T, and NK-like cell lines, as well as a MLC (Fig. 4a). RT-PCR products of $1,461,1,545$, and
Fig. 4 a RT-PCR analyses of IpLITR1 and IpLITR2 expression in various catfish clonal cell lines and a polyclonal MLC. Total RNA was obtained from the catfish 42TA macrophages, 3B11 B cells, TS32.15 and TS32.17 nonautonomous cytotoxic T cells, autonomous G14D T cells, a MLC, and 1F3 NK-like cells. RT-PCR was performed using primers specific for IpLITR1, IpLITR2, and IpEF1 $\alpha$. The sizes of the IpLITR bands verified by sequencing are indicated at the right margin and base pair sizes are at the left margin. $\mathbf{b}$ Schematic representation of IpLITR-types identified by sequencing of RT-PCR products obtained using IpLITR1 and IpLITR2-specific primers. The originally identified prototype IpLITRs are boxed, and arrows indicate the relative positions of the primer pairs used in RT-PCR reactions. Sizes in base pairs corresponding to the bands observed in a are indicated above each schematic. The predicted SP, Ig domains, TM, and CYT are indicated. ITIM-like motifs are shown as hatched boxes and noncanonical immunotyrosine-based activation motif-like motifs shown as white boxes within the CYT. Individual Ig domains are shaded according to relatedness as described for Fig. 1, and percentages above each receptor represent percentage of amino acid identities of the predicted extracellular region of the IpLITR-like sequences when compared with the prototype IpLITRs

2,392 bp were obtained using IpLITR1-specific primers, and these products were detected variably among the cell lines examined. It was also found that three RT-PCR products were also variably amplified from the different catfish leukocyte cell lines using IpLITR2-specific primers, the expected 780-bp product, a 1,298-bp product (previously observed in tissues; see Fig. 3c), and an additional 1,060-bp product (Fig. 4a). In contrast, no IpLITR message was detected in the fibroblast $\mathrm{CCO}$ cell line (data not shown), indicating that these genes are primarily expressed in cells of immune function.

The sequencing of the various IpLITR1 and IpLITR2 RTPCR products from all sources (both tissues and cell lines) resulted in the identification of multiple different IpLITRrelated sequences (Stafford et al. manuscript in preparation). Schematic diagrams of representative IpLITR1 and IpLITR2 variant forms are shown in Fig. 4b. These highly related IpLITR1- and IpLITR2-like sequences differed in the number of Ig-like domains present, and in the case of some IpLITR1-variants, also differed in the length of the CYT.

Homology between IpLITRs, LRC, and FcR genes

When the extracellular region of IpLITR1 was used in BLASTP analysis, various adhesion molecules, FcRs, FcR-like proteins (FCRLs), LILRs, and KIR gene family members were identified with significant homology (i.e., $\mathrm{E}$ values $<10^{-7}$ ), albeit with moderate amino acid identities ranging from $\sim 25$ $30 \%$ (Table 3). Examples include the adhesion molecules CD22 and carcinoembryonic antigen-related cell adhesion molecule 5, Xenopus leukocyte FCRLs, XFL, PIRA1, LILRA2, CHIRA2, KIR3DL1, FCRL5, and Fc $\gamma$ RIIB. When the Ig domains of IpLITR2 were used for screening, most 
a

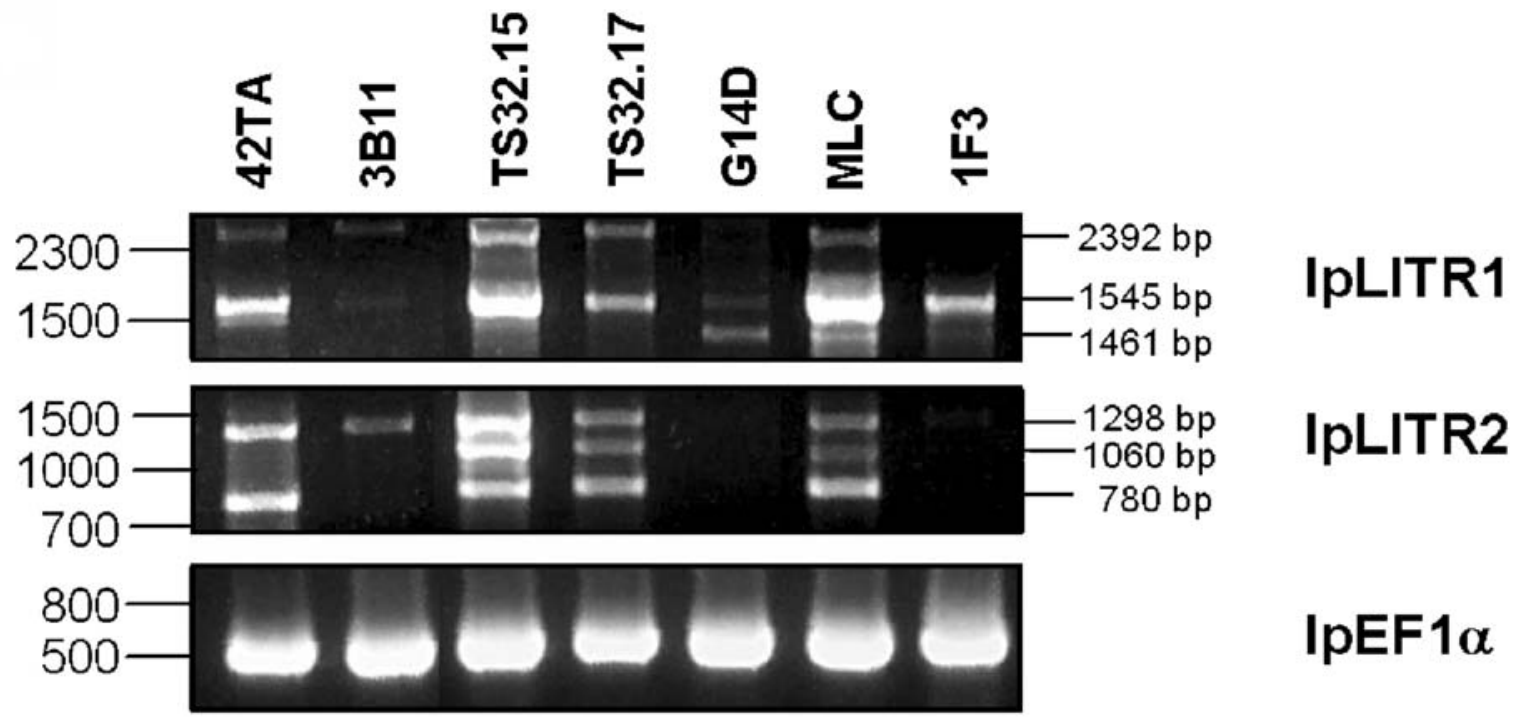

b

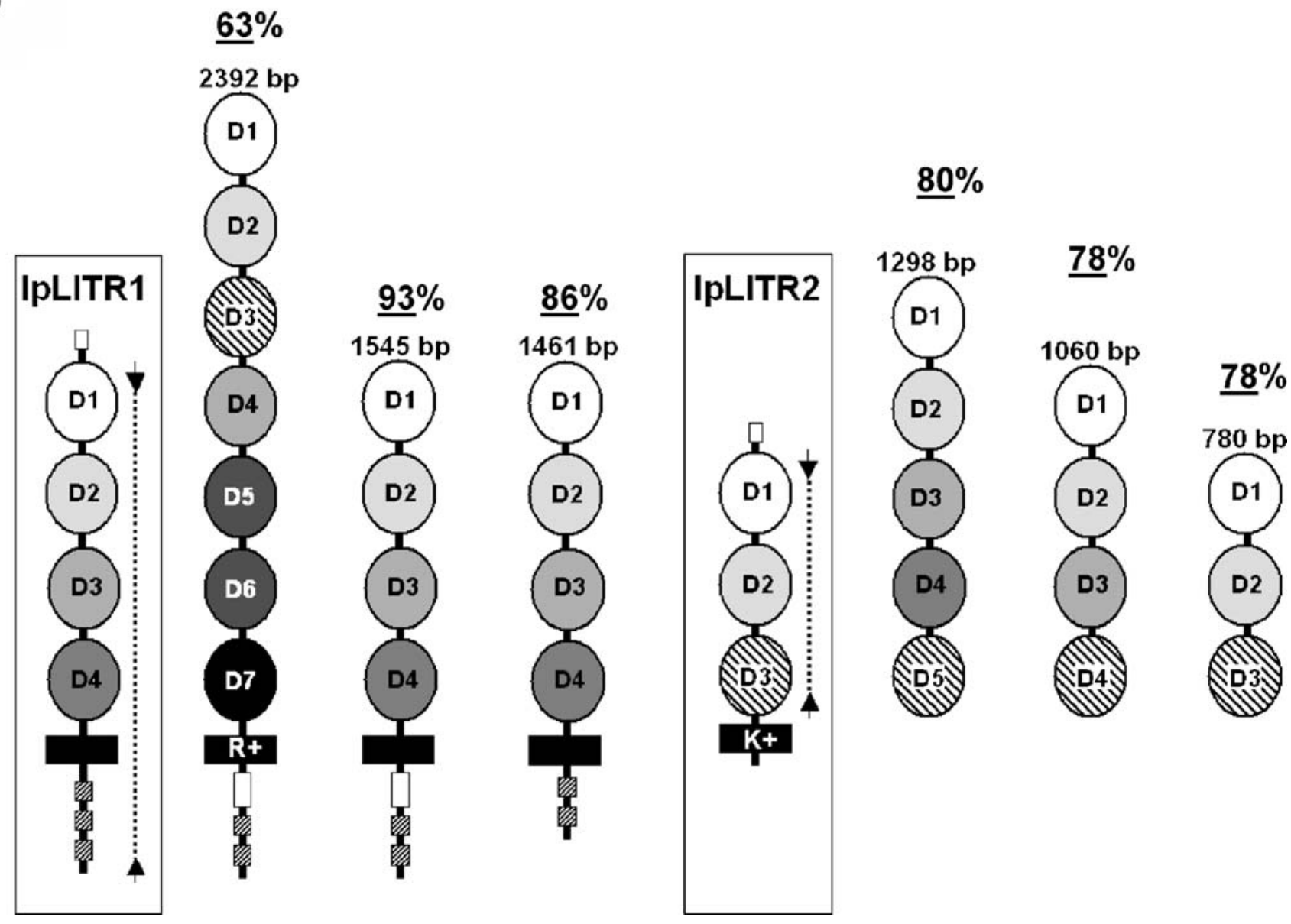

matches were members of the FcR gene family, e.g., Fc $\gamma$ RI, Fc $\gamma$ RII, Fc $\gamma$ RIII, and FceRI $\alpha$, from humans, primates, and rodents, as well as various FCRL proteins (Table 3). Searches using the extracellular region of IpLITR3 were essentially the same as the IpLITR1 BLASTP results, and PSI-BLAST analysis further reinforced the evolutionary relationships between IpLITRs and mammalian FcRs, FCRLs, KIRs, and LILRs, as well as avian CHIRs (data not shown). 
Phylogenetic analyses and alignments

The IpLITR D1 and D2 Ig domains are related to those of mammalian FcRs and FCRLs (Fig. 5a) in that these domains clustered with the FcR/FCRLs and away from catfish NITRs and from representative LRC members in a phylogenetic tree. In contrast to the D1/D2 sequence analyses, the membrane proximal D3 and D4 sequences of IpLITR1 and IpLITR3 resulted in a significant shift within the phylogenetic tree; IpLITRs now clustered with the LRC-encoded genes (Fig. 5b). The LRC relationship is supported by high bootstrap values when comparing IpLITR1 and IpLITR3 D3 and D4 sequences with D2 and D3 sequences of representative LILRs and KIRs, as well as with D1 and D2 sequences of NKp46 and CHIRs. The D5 and D6 sequences of IpLITR3 are related to the D1 and D2 domains of the FcR family (Fig. 5c). Thus, IpLITR3 is the best example of the unique domain structure of these teleost receptors, as it encodes for combinations of Ig domains that are phylogenetically related to the FcRs and FCRLs (D1 and D2; D5 and D6), as well as LRC encoded receptors (D3 and D4).

Subsequently, the D1 and D2 Ig domain sequences of IpLITR2 (as a representative) were aligned with various mouse and human FcRs and three representative human FCRLs. A majority of the predicted $\beta$-strands in IpLITR2 is present in similar positions as the $\beta$-strands found in FcR/FCRL family members (Fig. 6a), indicating a predicted degree of secondary structural conservation between IpLITRs and these mammalian immune receptors. In most cases, the predicted IpLITR2 $\beta$-strands were present in regions of high amino acid identity/similarity; however, none of the FcR residues known to contact the Fc portion of Ig were present (Fig. 6a). The D3 of IpLITR2, a domain specific to this IpLITR type, appears to be related to the membrane distal domain (D1) of several CHIRs (Fig. 6b), and alignment of IpLITR2 D3 with representative CHIR D1s indicated a degree of amino acid identity/conservation between these Ig domains (Fig. 6c). To reinforce the unique nature of domains present within this receptor, IpLITR2 D1 did not cluster with the CHIRs but was related to mammalian FcR D1 domains (Fig. 6b).

Attempts to model IpLITR Ig domains were unsuccessful as no sufficient modeling templates were identified using SWISS-Model. However, similarity searches against the NCBI's protein data bank (PDB) indicated a significant match between D1 and D2 of IpLITRs and the Ig domains of human FceRI $\alpha$ (E value $<10^{-8}$ ). Conversely, the membrane proximal D3 and D4 Ig domains of IpLITR1 and IpLITR3 had significant alignment scores (E value $<10^{-7}$ ) with the first two Ig domains of the human natural cytotoxicity receptor NKp46. Taken together, these PDB search results further support that IpLITRs D1 and D2 Ig domain sequences are related to mammalian FcRs and
FCRLs, and that the D3 and D4 Ig domains are related to LRC encoded receptors.

Mining of the zebrafish genome identified five contigs (Table 2) containing sequences encoding IpLITR-like proteins predicted to contain several Ig domains and, in some cases, signal peptides, TM segments, and CYTs with signaling motifs (Fig. 7). These findings indicate that IpLITR-like molecules are not unique to the catfish, but are likely common in other teleost species. Because IpLITR3 was identified as the closest match from the NCBI's database to the zebrafish (Zf) LITR-like proteins, it was used for further comparisons. Phylogenetic analysis of the various Ig domain sequences indicated that the ZfLITRlike proteins were closely related to those of IpLITRs (Fig. 7a). In most cases, the membrane proximal domains of ZfLITRs are highly related to their IpLITR3 counterparts with $>60 \%$ amino acid identity (Fig. 7b). However, one of the ZfLITRs (XP_692267) contains two Ig domains similar to IpLITR3 D6 in the membrane distal region. This zebrafish receptor also contains three Ig domains that are phylogentically related (Fig. 7a) with high amino acid identity $(\sim 50 \%)$ to IpLITR3 D4 (Fig. $7 \mathrm{~b})$. The other ZfLITR-like proteins are also composed of combinations of Ig domains that are related to IpLITR3 Ig domains with varying degrees of amino acid identities (e.g., 25-66\%). However, none of the predicted ZfLITR-like molecules contained membrane distal Ig domains that were related to IpLITR D1 and D2, which are shared among all the IpLITRs identified to date. This suggests either that LITR membrane distal domains may have coevolved with distinct ligands within individual fish species or that ZfLITRs with D1 and D2 domains similar to IpLITRs have yet to be identified. It should be noted, however, that these zebrafish domains were obtained from genomic sequence and that they do not accurately represent the precise organization of the Ig domains likely to be present in encoded ZfLITRs, which will only be determined from cDNA sequences. Nonetheless, the genomic segments are useful for domainto-domain comparisons and reinforced the presence of IpLITR-like sequences in another teleost species.

\section{Discussion}

The novel IpLITRs described herein represent a polymorphic and polygenic gene complex that are primarily expressed in hematopoietic tissues. The polymorphic nature of this gene complex was indicated by RFLP analysis, which revealed differences in where various restriction enzymes cut the genomic DNA among the sibling catfish. However, it should be noted that only detailed sequence analyses of these IpLITR genes in a number of individuals would be required to determine if any polymorphic 
Table 3 Representative IpLITR BLASTP results

\begin{tabular}{|c|c|c|c|c|c|}
\hline Receptor & & Accession & Score & E-value & Chromosome \\
\hline \multicolumn{6}{|l|}{ IpLITR1 } \\
\hline $\mathrm{CD} 22$ & Human & NP_001762 & 88 & $4 \mathrm{e}-16$ & $19 q 13.1$ \\
\hline XFL1.4 & Xenopus & AAQ56585 & 84 & $5 e-15$ & $?$ \\
\hline CEACAM5 & Human & CAA34474 & 84 & $5 e-15$ & $19 \mathrm{q} 13.1$ \\
\hline PIRA1 & Rat & AAD50905 & 73 & $1 \mathrm{e}-11$ & $?$ \\
\hline LILRA2 (ILT1) & Chimpanzee & NP_001009044 & 72 & $2 \mathrm{e}-11$ & 19 \\
\hline FCRL5 (FcRH5) & Mouse & NP_899045 & 71 & $6 e-11$ & 3 \\
\hline XFL1.3 & Xenopus & AAQ63874 & 71 & $6 \mathrm{e}-11$ & $?$ \\
\hline LILRA2 (ILT1) & Human & AAD50364 & 70 & $1 \mathrm{e}-10$ & $19 \mathrm{q} 13.4$ \\
\hline KIR3DL1 & Human & AAC 83928 & 69 & $3 e-10$ & $19 \mathrm{q} 13.4$ \\
\hline KIR & Chimpanzee & AAF86243 & 68 & $4 \mathrm{e}-10$ & 19 \\
\hline CHIRA2 & Chicken & CAG33731 & 66 & $1 \mathrm{e}-9$ & $?$ \\
\hline KIR3DL1 & Orangutan & AAM78465 & 66 & $1 e-9$ & 19 \\
\hline FCRL5 (FcRH5) & Human & CAH71428 & 66 & $2 e-9$ & 1q21 \\
\hline KIR3DS1 & Human & AAV32446 & 66 & $2 e-9$ & $19 \mathrm{q} 13.4$ \\
\hline Fc $\gamma$ RIIB & Rat & AAL29888 & 63 & $1 \mathrm{e}-8$ & $13 \mathrm{q} 24$ \\
\hline XFL1.6 & Xenopus & AAQ63873 & 63 & $1 \mathrm{e}-8$ & $?$ \\
\hline XFL1.2 & Xenopus & AAQ56587 & 62 & $2 \mathrm{e}-8$ & $?$ \\
\hline CHIRB3 & Chicken & CAG25957 & 62 & $3 e-8$ & $?$ \\
\hline LILRA3 (ILT6) & Human & AAB87661 & 60 & $8 \mathrm{e}-8$ & $19 \mathrm{q} 13.4$ \\
\hline LIR & Bovine & XP_586587 & 60 & $1 e-7$ & $?$ \\
\hline PIRA2 & Mouse & AAC53213 & 59 & $2 \mathrm{e}-7$ & 7 \\
\hline \multicolumn{6}{|l|}{ IpLITR2 } \\
\hline IRTA2 & Bovine & XP_595289 & 76 & $1 \mathrm{e}-12$ & $?$ \\
\hline Fc $\gamma$ RII & Guinea pig & A 34636 & 61 & $3 e-8$ & $?$ \\
\hline FCRL5 (FcRH5) & Mouse & NP_899045 & 60 & $6 e-8$ & 3 \\
\hline FCRL3 (FcRH3) & Mouse & AAS91578 & 60 & $6 e-8$ & 3 \\
\hline $\mathrm{Fc} \gamma \mathrm{RI}$ & Macaque & AAL92095 & 59 & $1 e-7$ & $?$ \\
\hline FCRL5 (FcRH5) & Human & NP_112571 & 57 & $4 e-7$ & $1 \mathrm{q} 21$ \\
\hline $\mathrm{Fc} \gamma \mathrm{RI} \alpha$ & Human & CAI 12557 & 57 & $5 e-7$ & $1 \mathrm{q} 21$ \\
\hline Fc $\gamma$ RIIB & Mouse & AAH19758 & 56 & $9 e-7$ & 1 \\
\hline XFL1.4 & Xenopus & AAQ56585 & 56 & $9 e-7$ & $?$ \\
\hline IRTA1 & Bovine & XP_614632 & 55 & $2 \mathrm{e}-6$ & $?$ \\
\hline FceRI $\alpha$ & Human & AA푸05912 & 53 & $8 \mathrm{e}-6$ & $1 \mathrm{q} 23$ \\
\hline FREB2 & Human & AAX11390 & 53 & $8 \mathrm{e}-6$ & $1 \mathrm{q} 23.3$ \\
\hline $\mathrm{Fc} \gamma \mathrm{RI}$ & Human & AAA58414 & 53 & $8 \mathrm{e}-6$ & $1 \mathrm{q} 21.2$ \\
\hline
\end{tabular}

Similarity search (BLASTP) of the NCBI's nonredundant protein database using the four Ig domains of IpLITR1 and the three Ig domains of IpLITR2

CEACAM5 carcinoembryonic antigen-related cell adhesion molecule, XFL Xenopus leukocyte FCRL

differences are also found within the coding regions. In addition, segregation analyses suggested that IpLITR genes are encoded within multiple independently segregating but homologous loci, similar to the situation seen with the ZfLITR homologs identified by genome mining. These paralagous relationships strongly suggest that IpLITRs are products of multiple gene duplication and translocation events from a common ancestoral gene. Whether or not the receptors encoded by the different IpLITR loci are functionally redundant remains to be determined. However, IpLITRs are related to functionally and genomically distinct mammalian FcRs and LRC encoded receptors, suggesting that such paralogous loci could have evolved to give rise to an array of immunoregulatory receptors with different functions.

The coordinate expression of IpLITRs by myeloid and lymphoid cell lines in combination with their potential activating and inhibitory signaling capabilities suggests that they play an important role in immune cell function(s). This possible importance of IpLITRs in immune responses is highlighted by the RT-PCR expression studies where a large number of highly related, but unique, IpLITR sequences were identified. These variant IpLITR sequences differed in the number of Ig domains present, and in some cases in the length of the CYT. Therefore, in addition to the prototype IpLITR1, IpLITR2, and IpLITR3 described here, 

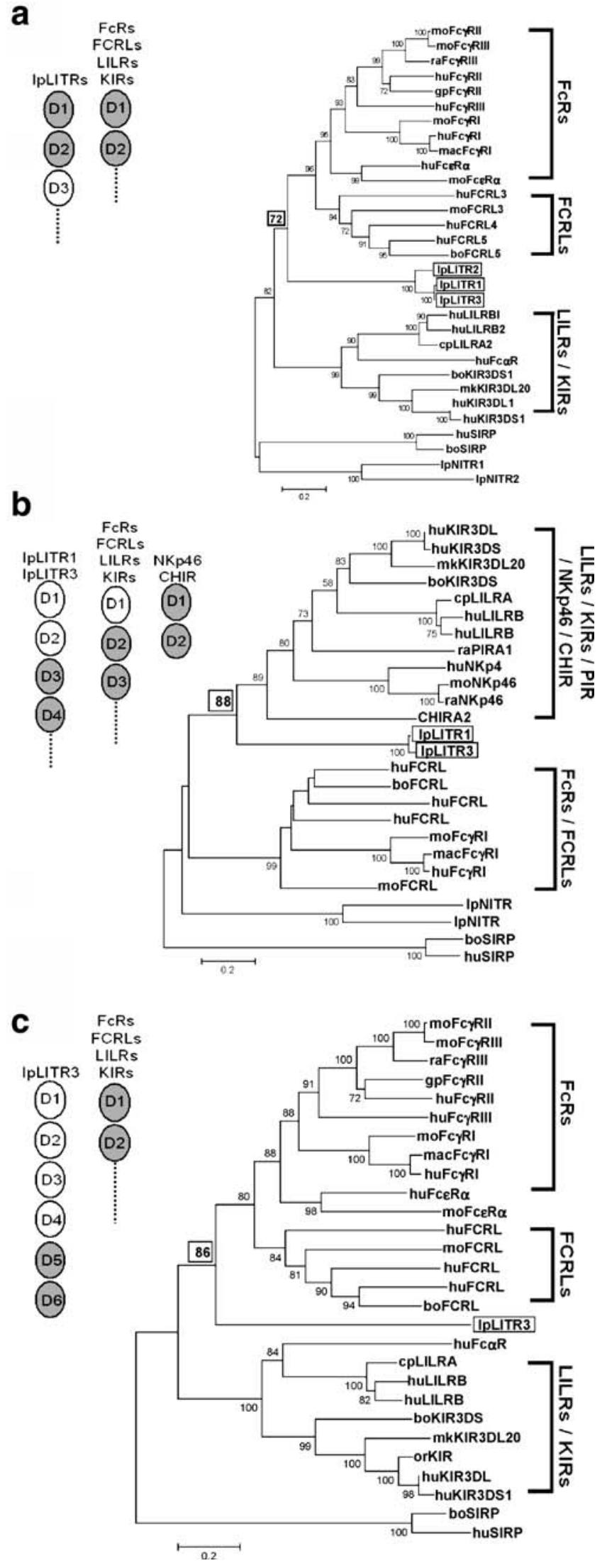

Fig. 5 Phylogenetic analyses reveal unique Ig domain composition within individual IpLITRs. The Ig domains compared are indicated by gray shading in the schematics to the left of each phylogenetic tree. a Comparisons of IpLITR, representative FcR and FCRL, and human LRC D1 and D2 sequences. b Comparisons of IpLITR1 D3 and D4 with representative FcR, FCRL, KIR, LILR, and PIRA1 D2 and D3 sequences, as well as NKp46 and CHIRA2 D1 and D2 sequences. c Comparison of IpLITR3 D5 and D6 with D1 and D2 of representative mammalian FcRs, FCRLs, and LILRs/KIRs. The accession numbers for the various Ig domain sequences used are: human (hu)Fc $\gamma$ RI(CAI12557), huFc $\gamma$ RII (CAA35642), huFc $\gamma$ RIII (CAA36870), huFceRI (AAH05912), huFCRL3 (AAH28933), huFCRL4 (AAK93970), huFCRL5 (NP_112571), huFc $\alpha$ R/CD89 (AAH27953), huLILRB1 (AAH15731), huLILRB2 (AAB87662), huKIR3DL1 (AAC83928), huKIR3DS1 (AAV32446), huSIRP (CAA71404), human NKp46 (AJ001383), mouse (mo)Fc $\gamma$ RI (AAD34931), moFc $\gamma$ RII (AAA37608), moFc $\gamma$ RIII (NP 034318), moFceRI (NP_034314), moFCRL3 (AAS91578), moNKp46 (AJ223765), rat (ra) Fc $\gamma$ RIII (AAA42050), raPIRA1 (XP 341773), raNKp46 (AF082533), guinea pig (gp) Fc $\gamma$ RII (D13692), macaque (mac)Fc $\gamma$ RI (AAL92095), bovine (bo)FCRL5 (XP_595289), boKIR3DS1 (AAP33626), boSIRP (CAA71943) chimpanzee (cp) LILRA2 (NP_001009044), orangutan (or)KIR (AAM78473), monkey (mk)KIR3DL20 (AAU50562), CHIRA2 (CAG33731), catfish (Ip) NITR1 (AF397454), and IpNITR2 (AF397455). For all analyses, SIRPs and/or IpNITRs were included as outgroups to root the trees. $\mathrm{NJ}$ trees with pairwise gap deletions were drawn using MEGA v3.0 (Kumar et al. 2001) with 10,000 bootstrap replications, and bootstrap values $>50 \%$ are shown. Branch lengths were measured in terms of amino acid substitutions, and scale bars are shown below the trees

other IpLITR family members are coexpressed by catfish myeloid and lymphoid cells. Whether or not some of the variously sized transcripts represent messages from different IpLITR genes or are the result of alternative splicing is unknown. However, the large number of unique IpLITRs found expressed suggests that IpLITRs represent a complex immune receptor family that can potentially generate a large number of IpLITR types.

Fig. 6 IpLITR2 contains Ig domains related to both FcRs/FCRLs and CHIRs. a Amino acid alignment of IpLITR2 D1 and D2 sequences with representative mammalian FcRs and FCRLs. Accession numbers are as in Fig. 5. Gray shading: residues similar/identical to IpLITR2. Boxed residues: contacts for Ig Fc in huFceRI. Black and gray arrows represent the predicted $\beta$-strands for IpLITR2 and huFcERI, respectively. Hatched boxes indicate conserved cyteines and dashes represent gaps. b Phylogenetic analysis of IpLITR2 D1 and D3 sequences (gray shaded) compared with representative mammalian FcR sequences (as in Fig. 5) and D1 sequences of representative CHIRs. The accession numbers for the various Ig domain sequences used are: CHIRB2 (XP_422905), CHIRB1 (CAH55757), CHIRAB2 (CAG33733), CHIRA2 (CAG33731), CHIRB5 (AJ879908), CHIR A1 (AF306851), CHIRB4 (XP_428342), CHIRB6 (CAI53861), and CHIRAB3 (AJ879909). NJ trees with pairwise gap deletions were drawn using MEGA v3.0 (Kumar et al. 2001) with 10,000 bootstrap replications, and bootstrap values $>50 \%$ are shown. Branch lengths were measured in terms of amino acid substitutions, and scale bars are shown below the trees. c Alignment of IpLITR2 D3 and CHIR D1. Gray shading indicates residues that are similar/identical to IpLITR2, black and gray arrows represent predicted $\beta$-strands for IpLITR 2 and CHIRA2, respectively; "dotted" lines indicate predicted helices and hatched boxes indicate conserved cyteines. Gaps in alignment are indicated by dashes 
The prototypical IpLITR1, IpLITR2, and IpLITR3 encode receptors with four, three, and six Ig domains, respectively. Pairwise comparisons and phylogenetic analysis of these domains suggest a high degree of conservation and a conserved membrane distal to membrane proximal ordering. Each of these three receptors has very similar D1 and D2 sequences, and IpLITR1 and IpLITR3 have almost identical
D3 and D4s. However, variation does occur between predicted membrane proximal Ig domains. The IpLITR2 D3 sequence is unique to this receptor and IpLITR3 D5 and D6 are not similar to any other of the prototypical IpLITR Ig domain sequences. Identification of LITR-like sequences on different zebrafish chromosomes and comparisons with IpLITRs supports the notion that these polymorphic and

a

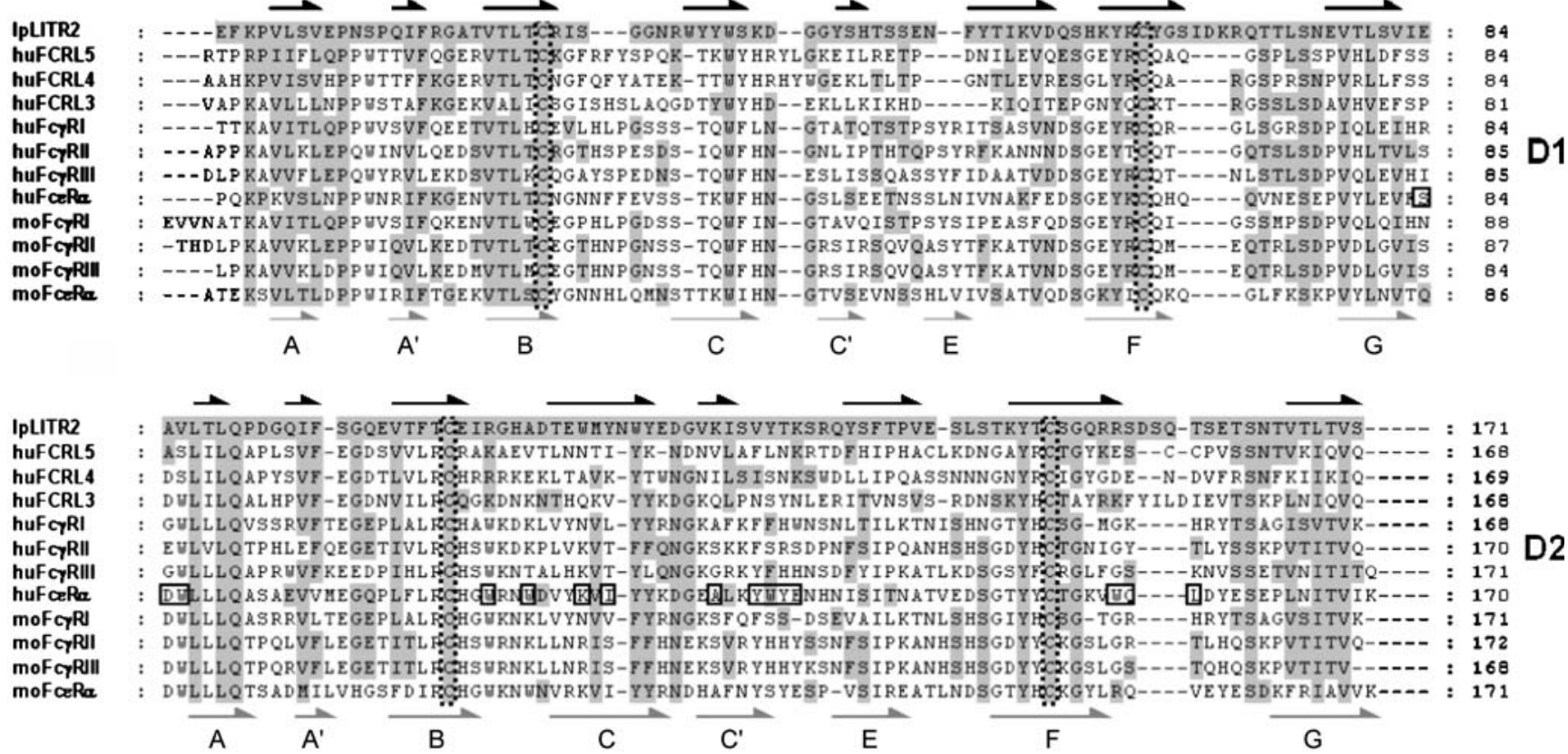

b
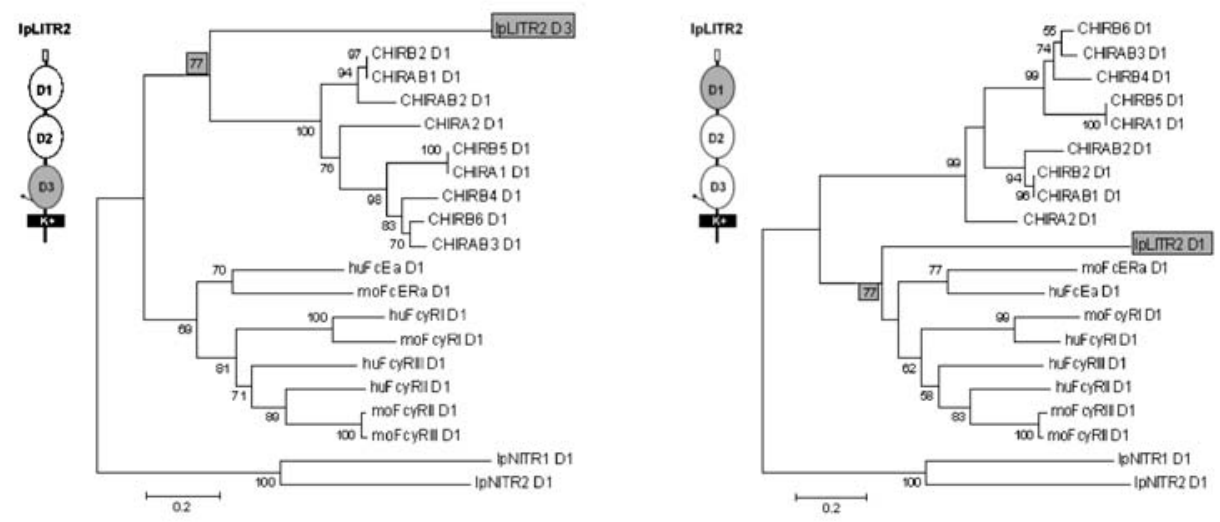

C 
polygenic immune receptors are not unique to catfish. Like IpLITRs, the predicted ZfLITRs are also composed of unique combinations of Ig domains. Such differences in the Ig domain compositions of IpLITRs and ZfLITRs may influence their ligand binding capabilities, formation of homo- or heterodimers and overall immunomodulatory functions.

IpLITR1 has a long CYT with two consensus ITIMs and a third ITIM-like motif, whereas both IpLITR2 and IpLITR3 have shorter CYTs devoid of recognizable signaling motifs. However, these latter two receptors each contain a lysine residue within their TM segments, indicating that associations with an adaptor molecule(s) would be necessary for them to initiate intracellular signaling (Daeron and Vivier 1999; Ravetch and Lanier 2000; Billadeau and Leibson 2002). Within the CYT of IpLITRI was also an overlapping ITSM suggesting that multiple motifs (ITIMs and ITSM) within the CYT of this receptor may participate in downstream signaling cascades through recruitment of various adaptor molecules (Sidorenko and Clark 2003). Similarly, predicted ZfLITR family members were shown to either contain long CYTs with ITIM motifs or short CYTs with charged TM segments. Genes for immunotyrosine-based-activation-motif-bearing adaptor molecules (e.g., $\mathrm{FcR} \gamma$ chain, $\mathrm{CD} 3 \zeta$, and DAP) have been identified in several ectothermic vertebrates, including frogs and fish (Guselnikov et al. 2003a,b). Hence, it seems likely that IpLITR2 and IpLITR3 would each associate with such molecules and function in a similar manner as mammalian receptors with charged TM segments. Thus, the paired expression pattern of IpLITRs with activatory and inhibitory signaling potential is very similar to that shown for several mammalian and avian families of immune receptors, including PIRs, LILRs, KIRs, FcRs, and CHIRs.

Similarity searches for IpLITR relatives revealed a striking dichotomy. The majority of matches were members of two distinct mammalian receptor families. While IpLITR2 appeared to be more closely related to FcRs and FCRLs, IpLITR1 and IpLITR3, exhibiting very similar D1-D4 sequences, were related to both FcR/FCRLs and LRC-encoded receptors. Thus, it is postulated that the $\mathrm{D} 1$ and $\mathrm{D} 2$ domains common to all IpLITRs are evolutionarily more closely related to domains encoded by the mammalian FcR and FCRLs, a contention supported by the retrieval of the human FceRI alpha chain as the most similar structure from a PDB search. Phylogenetic analyses also supported this hypothesis because the IpLITR D1 and D2 clustered with members of the FcR and FCRL receptor families and away from representative LRC gene family members. Although the D1D2 of IpLITRs are phylogenetically related to FcR and FcRLs, there is no evidence to support that IpLITRs bind Ig. It appears more likely that the relatedness of these membrane distal Ig domains, as also demonstrated for FCRLs, PIRs, and CHIRs, are only evident at the phylogenetic level and that they are not functional homologs. Furthermore, during the preparation of this manuscript, a "bona fide" Ig-binding catfish FcR homolog, which is not a member of the IpLITR receptor family, was identified and characterized (Stafford et al., 2006). In contrast to the D1 and D2 domains, IpLITR1 and IpLITR3 D3 and D4 Ig domains are related to several members of the LRC (human chromosome 19q13.4). As above, these two domains clustered with one family of receptors (the LRC) and not the other (i.e., FcR/FcRLs). The identification of NKp46 as a structural relative from the PDB also supports this notion. Interestingly, D5 and D6 of IpLITR3 are related to FcR and FCRLs, but their membrane proximal location suggests that they are unlikely to be involved in ligand recognition (i.e., Ig). Finally, the third domain of IpLITR2 clustered with the Ig domains of several representative CHIRs. Taken together, IpLITR extracellular domain sequences appear reminiscent of both FcR/FCRLs and members of the mammalian and avian LRC, implying that catfish LITRs are distant relatives to each of these genomically and functionally diverse receptor gene families. At present, the functional significance of these novel teleost immune receptors is unknown.

Recently, key insights into the evolution of the FcR and LRC gene families and their potential common origin has been presented: (1) human chromosomes 1 and 19 are believed to be in paralogous regions of the genome due to an entire genome duplication event (Kasahara 1999; Shiina et al. 2001); (2) attempts at cloning rodent $\mathrm{Fc} \alpha \mathrm{R}$ (CD89) orthologs identified the PIR gene family located on a mouse chromosome, which is syntenic to the human LRC (Kubagawa et al. 1997; Hayami et al. 1997); (3) the subsequent search for distant PIR relatives resulted in the discovery of the CHIRs, providing the first molecular evidence for a common ancestor of LRC and FcR gene families (Dennis et al. 2000); (4) sequencing of genomic segments encoding the CHIR gene family identified the chicken equivalent of an LRC that appears to have also been expanded by gene duplication events (Nikolaidis et al. 2005a); and (5) the identification of Iglike domains in chickens, Xenopus, and bony fish that are related to Ig domains of mammalian FcRs and members of the LRC (Nikolaidis et al. 2005b). Taken together, these findings suggest that immune receptors encoded by human chromosome 1q21 and 19q13 most likely evolved from a common ancestor that has since undergone multiple rounds of duplication and homologous recombination events.

Although there is functional evidence for the existence of FcR- or KIR/LILR-like homologs in ectothermic vertebrates (Coosemans and Hadji-Azimi 1986; Shen et al. 2003, 2004), their genetic identification has not been previously reported and the inability to clone these receptors has made it difficult to elucidate their evolutionary history. The recent discovery of mammalian FCRL genes located on chromosome 1q21-22, however, has expanded the view of immune receptor evolution (Davis et al. 2001; Hatzivassiliou et al. 
a

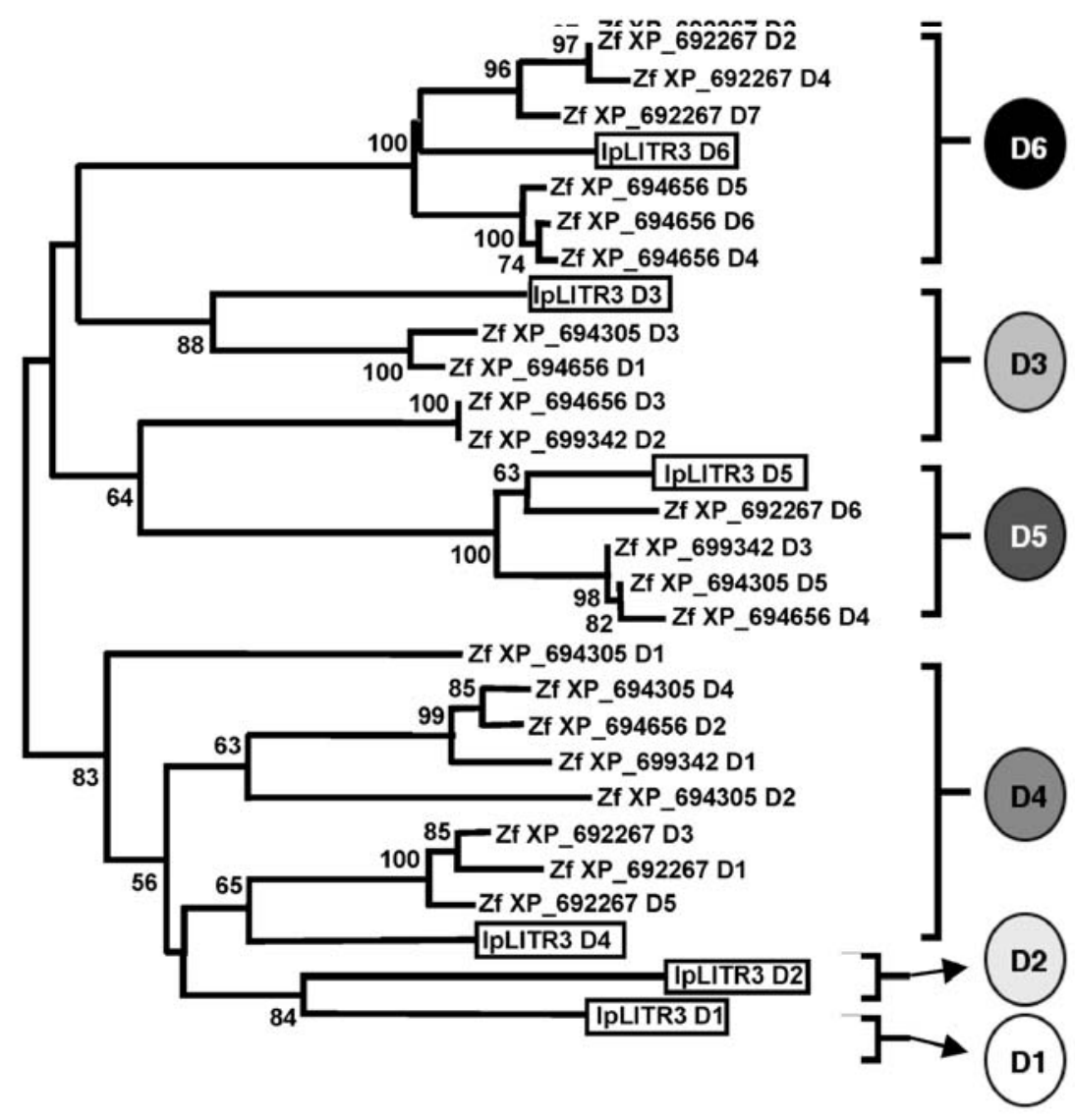

b

IpLITR3

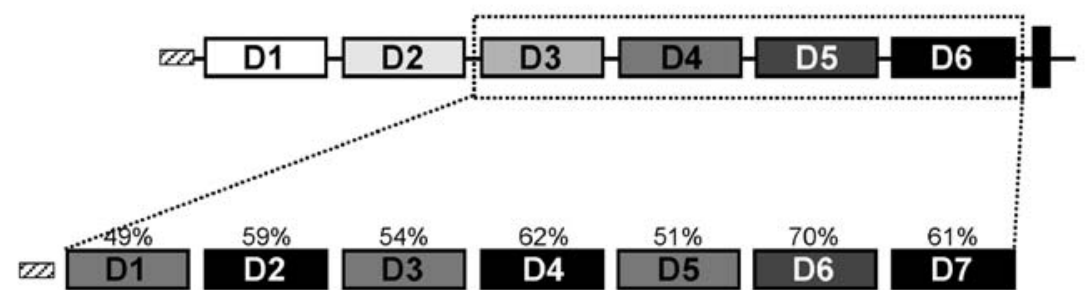

531 aa

Zf XP_692267 Chr. 3

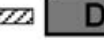

D2

D4

D5

D7

833 aa

Zf XP_694305 Chr. 8

\begin{tabular}{ll}
$39 \%$ & $39 \%$ \\
\hline $\mathrm{D} 1$ & $\mathrm{D} 2$ \\
\hline
\end{tabular}

$32 \%$

$48 \%$

$65 \%$

$62 \%$

D5 D6

D6 $= \pm=$

745 aa

Zf XP_694656 Chr. 8

\begin{tabular}{l}
$35 \%$ \\
\hline D1 \\
\hline
\end{tabular}

$51 \%$ $33 \%$

D3

$60 \%$

\section{$66 \%$}

D5

\section{Zf XP_699342 Chr. ?}

\begin{tabular}{llll}
$25 \%$ & $33 \%$ & $64 \%$ & $61 \%$ \\
\hline$D 1$ & D2 & D3 & D4
\end{tabular}

461 aa

Fig. 7 Annotation of zebrafish LITR-like proteins. a Phylogenetic analysis of ZfLITR and IpLITR3 Ig domains. Ig domains were predicted using SMART (Letunic et al. 2004) and the amino acid sequences aligned using CLUSTALW (Thompson et al. 1997). NJ trees with pairwise gap deletions were drawn using MEGA v3.0 (Kumar et al. 2001). Only branch values $>50$ are shown, and the major Ig domain groups are designated by brackets and shaded according to their relatedness with IpLITR3 Ig domains. b Schematic representation of ZfLITR-like proteins compared with IpLITR3. Domains are shaded according to their phylogenetic relationship with IpLITR3 domains. Hatched bars on IpLITR3 and Zf XP_692267 indicate signal peptides, TM segments are solid black lines, and ITIMs are shown as gray boxes for XP_694305. Amino acid identity vs corresponding IpLITR3 Ig domains are indicated as percentage value above each domain, and only IpLITR3 D3, D4, D5, and D6 were related to the Ig domains of the representative ZfLITR analyzed, as indicated by the dashed box. Predicted size in amino acids for each molecule is indicated on the left 
2001; Miller et al. 2002; Guselnikov et al. 2002). For example, FCRL3, FCRL4, and FCRL5 not only encode D1, D2, and D3 Ig domains homologous to the corresponding domains of Fc $\gamma$ RI, but also contain variable numbers of unique membrane proximal Ig domains (Davis et al. 2001; Hatzivassiliou et al. 2001). Homology searches demonstrated that the FCRLs, also termed IFGP (for IgSF, FcR, and gp42) exhibited a "chimeric" Ig domain compositions and consequently these receptors were proposed to be a phylogenetic link between the FcRs, rat NK cell-specific gp42 antigen, and adhesion molecules (Guselnikov et al. 2002). This feature of similar Ig domains encountered in homologous but functionally diverse receptors is also a striking feature of the IpLITRs and supports the hypothesis that certain mammalian immune receptor families (i.e., FcRs and KIRs) have evolved from a common ancestor. Notably, although the IpLITRs D1 and D2 domains are phylogenetically and structurally similar to the mammalian FcRs and FCRLs, the IpLITR ligand(s) is unknown. This is similar to the situation with mammalian FCRL molecules, whose membrane distal Ig domains are clearly related to the classic FcRs, but are unlikely to be functional homologs because they do not bind Ig (Hatzivassiliou et al. 2001; Davis et al. 2005). Therefore, although certain Ig domains encoded by several receptor gene families identified in humans, rodents, birds, and fish (i.e., FCRLs, PIRs, CHIRs, and IpLITRs, respectively) are related to $\mathrm{FcRs}$, they appear to have evolved to recognize different ligands. Recently, it was shown that rodent PIRs recognized MHC class 1 and $\beta_{2} \mathrm{M}$ (Takai 2005), raising the possibility that related immunoregulatory receptors in other species (i.e., CHIRs and IpLITRs) could also be involved in MHC recognition.

In summary, the discovery of individual catfish receptors that encode Ig domains related to both FcR and LRC gene families provides further support for the common origin of these important mammalian immune receptors. Identification of LITR-like sequences in the zebrafish genome also demonstrates that these receptors are likely found in all teleosts. Annotation of these genomic segments will be necessary to determine if zebrafish LITR homologs exhibit conserved genomic features and syntenic genomic location with mammalian 1q21-23 and/or 19q13. Thus, the discovery of IpLITRs is an important step towards further understanding the common origin and evolutionary history of the different families of mammalian immunoregulatory receptors. The investigation of the functional significance of these novel teleost receptors is underway and will be aided by the availability of channel catfish cell lines that coordinately express IpLITRs and the eventual production of IpLITR specific antibodies.
Acknowledgements This work was supported by grants from the National Institutes of Health (RO1AI-19530), National Sciences Foundation (MCB-0211785), US Department of Agriculture (2002-3520412211), and a Natural Sciences and Engineering Research Council of Canada postdoctoral fellowship to J.L. Stafford. We thank Cecile Snell for skillful technical assistance and Dr. Chinchar for the CCO cell line.

\section{References}

Altschul SF, Gish W, Miller W, Meyers EW, Lipman DJ (1990) Basic local alignment search tool. J Mol Biol 215:403-410

Bateman A, Coin L, Durbin R, Finn RD, Hollich V, Griffiths-Jones S, Khanna A, Marshall M, Moxon S, Sonnhammer EL, Studholme DJ, Yeats C, Eddy SR (2004) The Pfam protein families database. Nucleic Acids Res 32:D138-D141

Billadeau DD, Leibson PJ (2002) ITAMs versus ITIMs: striking a balance during cell regulation. J Clin Invest 109:161-168

Browser PR, Plumb JA (1980) Fish cell lines: establishment of a line from ovaries of channel catfish. In Vitro 16:365-368

Coosemans V, Hadji-Azimi I (1986) Partial characterization of different cell types found in the Xenopus laevis lymphoreticular tumor based on the presence or absence of surface immunoglobulins and Fc molecules. Dev Comp Immunol 10:547-549

Daeron M (1997) Fc receptor biology. Annu Rev Immunol 15:203-234

Daeron M, Vivier E (1999) Biology of immunoreceptor tyrosine-based motif-bearing molecules. Curr Top Microbiol Immunol 244:1-12

Davis RS, Wang YH, Kubagawa H, Cooper MD (2001) Identification of a family of $\mathrm{Fc}$ receptor homologs with preferential B cell expression. Proc Natl Acad Sci U S A 98:9772-9777

Davis S, Ehrhardt GRA, Leu CM, Hirano M, Cooper MD (2005) An extended family of Fc receptor relatives. Eur J Immunol 35:674-680

Dennis G Jr, Kubagawa H, Cooper MD (2000) Paired Ig-like receptor homologs in birds and mammals share a common ancestor with mammalian Fc receptors. Proc Natl Acad Sci U S A 29:30-37

Falk N, Ravetch RV (2006) Fc $\gamma$ receptors: old friends and new family members. Immunity 24:19-28

Falk N, Bruhns P, Horiuchi K, Ravetch RV (2005) Fc $\gamma$ RIV: a novel FcR with distinct IgG subclass specificity. Immunity 23:41-51

Guselnikov SV, Ershova SA, Mechetina LV, Najakshin AM, Volkova OY, Alabyev BY, Taranin AV (2002) A family of highly diverse human and mouse genes structurally links leukocyte FcR, gp42 and PECAM-1. Immunogenetics 54:87-95

Guselnikov SV, Bell A, Najakshin AM, Robert J, Taranin AV (2003a) Signaling FcR $\gamma$ and TCR $\zeta$ subunit homologs in the amphibian Xenopus laevis. Dev Comp Immunol 27:727-733

Guselnikov SV, Najakshin AM, Taranin AV (2003b) Fugu rubripes possesses genes for the entire set of the ITAM-bearing transmembrane signal subunits. Immunogenetics 55:472-479

Hatzivassiliou G, Miller I, Takizawa J, Palanisamy N, Rao PH, Lida S, Tagawa S, Taniwaki M, Russo J, Neri A, Cattoretti G, Clynes R, Mendelsohn C, Chaganti RS, Dalla-Favera R (2001) IRTA1 and IRTA2, novel immunoglobulin superfamily receptors expressed in B cells and involved in chromosome 1q21 abnormalities in B cell malignancy. Immunity 14:277-289

Hawke NA, Yoder JA, Haire RN, Mueller MG, Litman RT, Miracle AL, Stuge T, Shen L, Miller N, Litman GW (2001) Extraordinary variation in a diversified family of immune-type receptor genes. Proc Natl Acad Sci U S A 98:13832-13837

Hayami K, Fukuta D, Nishikawa Y, Yamashita Y, Inu M, Ohyama Y, Hikida M, Ohmori H, Takai T (1997) Molecular cloning of a novel 
cell-surface glycoprotein homologous to killer cell inhibitory receptors. J Biol Chem 272:7320-7327

Hogan RJ, Waldbieser G, Goudie CA, Antao A, Godwin UB, Wilson MR, Miller NW, Clem LW, McConnell TJ, Wolters WR, Chinchar VG (1999) Molecular and immunological characterization of gynogenetic channel catfish (Ictalurus punctatus). Mar Biotechnol (NY) 1:317-327

Hulett MD, Hogarth PM (1994) Molecular basis for Fc receptor function. Adv Immunol 57:1-127

Hulett MD, Osman N, McKenzie IF, Hogarth, PM (1991) Chimeric Fc receptors identify functional domains of the murine high affinity receptor for IgG. J Immunol 147:1863-1868

Kasahara M (1999) The chromosomal duplication model of the major histocompatibility complex. Immunol Rev 167:17-32

Kelly LA, MacCallum RM, Sternberg MJ (2000) Enhanced genome annotation using structural profiles in the program 3D-PSSM. J Mol Biol 299:499-520

Kremer EJ, Kalatzis V, Baker E, Callen DF, Sutherland GR, Maliszewski CR (1992) The gene for the human IgA Fc receptor maps to $19 \mathrm{q} 13.4$. Hum Genet 89:107-108

Kubagawa H, Burrows P, Cooper MD (1997) A novel pair of immunoglobulin-like receptors expressed by B cells and myeloid cells. Proc Natl Acad Sci U S A 94:5261-5266

Kumar S, Tamura K, Jakobsen IB, Nei M (2001) MEGA2: molecular evolutionary genetics analysis software. Bioinformatics 17:1244-1245

Letunic I, Copley RR, Schmidt S, Ciccarelli FD, Doerks T, Schultz J, Ponting CP, Bork P (2004) SMART 4.0: towards genomic data integration. Nucleic Acids Res 32:D142-D144

Martin AM, Kulski JK, Witt C, Pontarotti P, Christiansen FT (2002) Leukocyte Ig-like receptor complex (LRC) in mice and men. Trends Immunol 23:81-88

Miller NW, Rycyzyn MA, Wilson MR, Warr GW, Naftel JP, Clem LW (1994a) Development and characterization of channel catfish long term B cell lines. J Immunol 152:2180-2189

Miller NW, Chinchar VG, Clem LW (1994b) Development of leukocyte cell lines from the channel catfish (Ictalurus punctatus). J Tissue Cult Methods 16:117-123

Miller I, Hatzivassiliou G, Cattoretti G, Madelsohn C, Dalla-Favera R (2002) IRTAs: a new family of immunoglobulin-like receptors differentially expressed in B cells. Blood 99:2662-2669

Nikolaidis N, Makalowska I, Chalkia D, Makalowski W, Klein J, Nei M (2005a) Origin and evolution of the chicken leukocyte receptor complex. Proc Natl Acad Sci U S A 102:4057-4062

Nikolaidis N, Klein J, Nei M (2005b) Origin and evolution of the Ig-like domains present in mammalian leukocyte receptors: insights from chicken, frog, and fish homologues. Immunogenetics 57:151-157

Quiniou SM, Wilson M, Bengten E, Waldbieser GC, Clem LW, Miller NW (2005) MHC RFLP analyses in channel catfish full-sibling families: identification of the role of MHC molecules in spontaneous allogeneic cytotoxic responses. Dev Comp Immunol 29:457-467

Ravetch JV, Kinet JP (1991) Fc receptors. Annu Rev Immunol 9:457-492

Ravetch JV, Lanier LL (2000) Immune inhibitory receptors. Science 290:84-89

Shen L, Stuge TB, Zhou H, Khayat M, Barker KS, Quiniou SMA, Wilson M, Bengten E, Chinchar VG, Clem LW, Miller NW (2002) Channel catfish cytotoxic cells: a mini-review. Dev Comp Immunol 26:141-149

Shen L, Stuge TB, Evenhuis JB, Bengten E, Wilson M, Chinchar VG, Clem LW, Miller NW (2003) Channel catfish NK-like cells are armed with IgM via a putative Fc $\mu$ R. Dev Comp Immunol 27:699-714

Shen L, Stuge TB, Bengten E, Wilson M, Chinchar VG, Naftel JP, Bermanke JM, Clem LW, Miller NW (2004) Identification and characterization of clonal NK-like cells from channel catfish (Ictalurus punctatus). Dev Comp Immunol 28:139-152

Shiina T, Ando A, Suto Y, Kasai F, Shigenari A, Takishima N, Kikkawa
E, Iwata K, Kuwano Y, Kitamura Y, Matsuzawa Y, Sano K, Nogami M, Kawata H, Li S, Fukuzumi Y, Yamazaki M, Tashiro H, Tamiya G, Kohda A, Okumura K, Ikemura T, Soeda E, Mizuki N, Kimura M, Bahram S, Inoko H (2001) Genomic anatomy of a premier major histocompatibility complex paralogous region on chromosome 1q21-q22. Genome Res 11:789-802

Shlapatska LM, Mikhalap SV, Berdova AG, Zelensky OM, Yun TJ, Nichols KE, Clark EA, Sidorenko SP (2001) CD150 association with either the SH2-containing inositol phosphatase or the SH2containing protein tyrosine phosphatase is regulated by the adaptor protein SH2D1A. J Immunol 166:5480-5487

Sidorenko SP, Clark EA (2003) The dual-function CD150 receptor subfamily: the viral attraction. Nat Immunol 4:19-24

Stafford JL, Wilson M, Nayak D, Quiniou SM, Clem LW, Miller NW, Bengtén W (2006) Identification and characterization of a FcR homolog in an ectothermic vertebrate, the channel catfish (Ictalurus punctatus). J Immunol (in press)

Strong SJ, Mueller MJ, Litman RT, Hawke NA, Haire RN, Miracle AL, Rast JP, Amemiya CT, Litman GW (1999) A novel multigene family encodes diversified variable regions. Proc Natl Acad Sci U S A 96:15080-15085

Stuge TB, Yoshida SH, Chinchar VG, Miller NW, Clem LW (1997) Cytotoxic activity generated from channel catfish peripheral blood leukocytes in mixed leukocyte cultures. Cell Immunol 177:154-161

Stuge TB, Wilson MR, Zhou H, Barker KS, Bengten E, Chinchar VG, Miller NW, Clem LW (2000) Development and analysis of various clonal alloantigen-dependent cytotoxic cell lines from channel catfish. J Immunol 164:2971-2977

Takai T (2005) A novel recognition system for MHC class I molecules constituted by PIR. Adv Immunol 88:161-192

Thompson JD, Gibson TJ, Plewniak F, Jeanmougin F, Higgins DJ (1997) The CLUSTAL_X windows interface: flexible strategies for multiple sequence alignment aided by quality analysis tools. Nucleic Acids Res 24:4876-4882

van Ginkel FW, Miller NW, Lobb CJ, Clem LW (1992) Characterization of anti-hapten antibodies generated in vitro by channel catfish peripheral blood lymphocytes. Dev Comp Immunol 161:39-151

Viertlboeck BC, Habermann FA, Schmitt R, Groenen MAM, Du Pasquier LD, Gobel TW (2005) The chicken leukocyte receptor complex: a highly diverse multigene family encoding at least six structurally distinct receptor types. J Immunol 175:385-393

Wagtmann N, Rojo S, Eichler E, Mohrenweiser H, Long EO (1997) A new human gene complex encoding the killer cell inhibitory receptors and related monocyte/macrophage receptors. Curr Biol 7:615-618

Wende H, Colonna M, Ziegler A, Volz A (1999) Organization of the leukocyte receptor cluster (LRC) on human chromosome 19q13.4. Mamm Genome 10:154-160

Wilson M, Bengten E, Miller NW, Clem LW, Du Pasquier L, Warr GW (1997) A novel chimeric Ig heavy chain from a teleost fish shares similarities to IgD. Proc Natl Acad Sci U S A 94:4593-4597

Yoder JA (2004) Investigating the morphology, function and genetics of cytotoxic cells in bony fish. Comp Biochem Physiol C Toxicol Pharmacol 138:271-280

Yoder JA, Mueller MG, Wei S, Corliss BC, Prather DM, Willis T, Litman RT, Djeu JY, Litman GW (2001) Immune-type receptor genes in zebrafish share genetic and functional properties with genes encoded by the mammalian leukocyte receptor cluster. Proc Natl Acad Sci U S A 98:6771-6776

Yoder JA, Mueller MG, Nichols KM, Ristow SS, Thorgaard GH, Ota T, Litman GW (2002) Cloning novel immune-type inhibitory receptors from the rainbow trout, Oncorhynchus mykiss. Immunogenetics 54:662-670 